PREPARED FOR SUBMISSION TO JINST

$11^{\text {Th }}$ International Conference on Position Sensitive Detectors

$3^{\text {RD }}-8^{\text {TH }}$ SEPTEMBER 2017

The Open University, Milton Keynes, MK7 6AA, England

\title{
A new strips tracker for the upgraded ATLAS ITk detector
}

\section{David, on behalf of the ATLAS Collaboration}

Deutsches Elektronen-Synchrotron (DESY),

Noktestr. 85, 22607, Hamburg, Germany

E-mail: claire.david@desy.de 
Aвstract: The inner detector of the present ATLAS detector has been designed and developed to function in the environment of the present Large Hadron Collider (LHC). At the next-generation tracking detector proposed for the High Luminosity LHC (HL-LHC), the so-called ATLAS Phase-II Upgrade, the particle densities and radiation levels will be higher by as much as a factor of ten. The new detectors must be faster, they need to be more highly segmented, and covering more area. They also need to be more resistant to radiation, and they require much greater power delivery to the front-end systems. At the same time, they cannot introduce excess material which could undermine performance. For those reasons, the inner tracker of the ATLAS detector must be redesigned and rebuilt completely. The design of the ATLAS Upgrade inner tracker (ITk) has already been defined. It consists of several layers of silicon particle detectors. The innermost layers will be composed of silicon pixel sensors, and the outer layers will consist of silicon microstrip sensors. This contribution focuses on the strip region of the ITk. The central part of the strips tracker (the "barrel") will be composed of rectangular "short" $(\sim 2.5 \mathrm{~cm})$ and "long" $(\sim 5 \mathrm{~cm})$ strip sensors. The forwards regions of the strips tracker (the "endcaps") consist of six disks per side, with trapezoidal shaped microstrip sensors of various lengths and strip pitches. In response to the needs of the strip region for the ITk, highly modular structures are being studied and developed, called "staves" for the central region (barrel) and "petals" for the forward regions (end-caps). These structures integrate large numbers of sensors and readout electronics, with precision light weight mechanical elements and cooling structures. The silicon sensors are fabricated in n-in-p float zone (FZ) technology. Low mass kapton-based circuit boards (the "hybrids") are directly glued on top of the sensors, hosting the so-called ABCN130 binary readout ASICs. Those ASICs are fabricated in a $130 \mathrm{~nm}$ CMOS process. The ASICs are connected to the microstrips via wirebonds. There are 256 channels per ABCN130. Those silicon "modules" are then directly glued onto low-mass, carbon fiber-based stave and petal core structures, with embedded titanium cooling pipes and data and power rails. A data concentrator board on each stave and petal side (the "end of structure" board, EoS) sends all the multiplexed data to the outside world via optical links, and host most of the components of the Detector Control System (DCS). The staves and petals are then arranged into cylinders and disks, respectively, by means of the integration and global structures. The service module elements, part of the global structure, provide data, power, and cooling to groups of petals and staves. In the baseline design, each of the service modules provides services to eight staves/petals. A strong prototyping effort has been put in place over the course of the last years in order to optimise the stave and petal structures. This contribution summarises the R\&D activities performed by the numerous institutes within the Strips ITCERN-LHCC-2017-005. ATLAS-TDR-025k collaboration that culminated recently in the release of the ATLAS Strips ITk Technical Design Report (TDR).

KEYWORDs: Large detector systems for particle and astroparticle physics; Particle tracking detectors; Radiation-hard detectors; Si microstrip and pad detectors 


\section{Contents}

1 Introduction 1

2 Overview of the ATLAS Upgrade inner tracker 2

2.1 Sensor technology 2

2.2 Strip module concept 3

2.3 Signals and electronics 3

2.4 Module support 5

3 Research and development $\quad 7$

$\begin{array}{lll}3.1 & \text { Total Ionizing Dose (TID) current increase } & 7\end{array}$

$\begin{array}{lll}3.2 & \text { Testbeam results } & 8\end{array}$

$\begin{array}{lll}3.3 & \text { Prototype characterisation } & 9\end{array}$

4 Conclusion $\quad 10$

\section{Introduction}

The ATLAS detector [1] at the Large Hadron Collider [2] is a general purpose particle detector investigating interactions at the subatomic level. In 2016, ATLAS recorded more than 35 inverse femtobarns ${ }^{1}$ of proton-proton collisions at 13 Tera electrovolts and is expected to collect 150 by the end of 2018. The current ATLAS Inner Tracking Detector has been designed to function at a constant instantaneous luminosity of $\mathcal{L}=1.0 \times 10^{34} \mathrm{~cm}^{-2} \mathrm{~s}^{-1}$. To fully exploit the potential of a machine as unique as the Large Hadron Collider (LHC) complex, a drastic luminosity increase is needed. This would make it possible to significantly extend the statistical sensitivity to new physics, as well as to maximise the performance for precision measurements. The proposed High Luminosity LHC project (HL-LHC), scheduled to start in 2026, plans to increase the total number of collisions by a factor of 10 beyond the original design value (from 300 to $3000 \mathrm{fb}^{-1}$ ).

As a consequence of the increased luminosity, the next generation of ATLAS components need to withstand higher levels of fluences in order to function with peak luminosities of 5.0 $7.5 \times 10^{34} \mathrm{~cm}^{-2} \mathrm{~s}^{-1}$. This new version of detector, referred to as the ATLAS Phase-II Upgrade, has to meet higher speed and radiation hardness requirements. The current ATLAS Inner Tracking Detector will be unable to cope with these extreme data rates and the high fluences for several reasons. The sensors and electronics were designed to withstand radiation damage that is equivalent to integrated luminosities from 400 to $850 \mathrm{fb}^{-1}$ only. Moreover, the data volume demands a new trigger design, which the current front-end electronics cannot achieve due to speed and bandwidth limitations. Also, some of the current systems will reach 100\% occupancy in such a dense environment, which can be compensated by increasing the readout surface areas and granularity.

\footnotetext{
${ }^{1}$ One inverse femtobarn $\left(\mathrm{fb}^{-1}\right)$ corresponds to approximately 100 trillion $\left(10^{12}\right)$ proton-proton collisions.
} 
Because of these limitations compromising the physics reach and performance, the current ATLAS Inner Tracking Detector will be fully replaced by an all-silicon inner tracker, the so-called ITk.

\section{Overview of the ATLAS Upgrade inner tracker}

The new Inner Tracker (ITk) consists of the Pixel Detector closest to the interaction point, surrounded by the Strip Detector. Each element is further divided into one cylinder-shaped "barrel" around the interaction point parallel to the beam pipe, closed by two "end-caps" on each side. The Pixel Detector counts more than 580 million electronics channels with a total silicon surface area of $13 \mathrm{~m}^{2}$. The Strip Detector has roughly 60 million channels and an area of silicon sensors of $165 \mathrm{~m}^{2}$, which is 2.5 times more than the current Inner Detector silicon strip system. It covers \pm 2.7 units of rapidity, which corresponds to an angle of about $15^{\circ}$ with respect to the beam axis. Figure 1

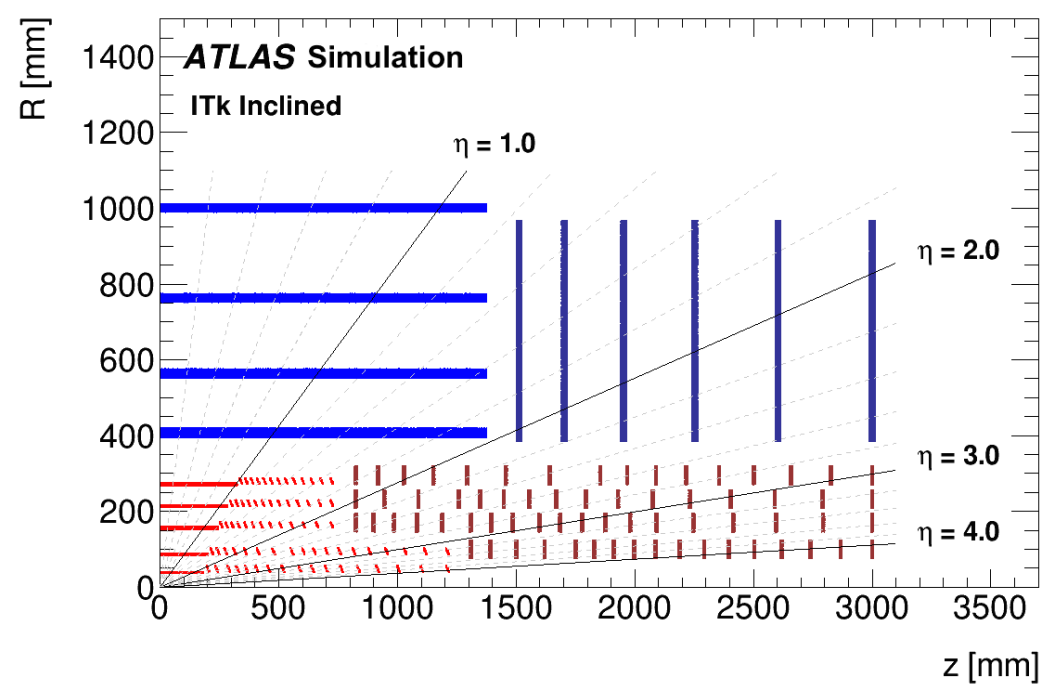

Figure 1: Schematic layout of the ITk for the HL-LHC phase of ATLAS. Here only the upper right quadrant is shown, with the interaction point at zero and the beam line aligned along the horizontal axis. The vertical axis is the radius, measured from the interaction point. The Pixel Detector elements are in red, while the Strip Detector parts are shown in blue. The barrel layers correspond to the horizontal elements and the end-cap disks are shown on the right with vertical lines [3].

shows a schematic layout of the ITk, with the Pixel and Strip Detector components in red and blue respectively. The following sections describe the Strip Detector, from the sensor technology to its global infrastructure. More details are available in the ATLAS Strips ITk Technical Design Report [3], published in April 2017.

\subsection{Sensor technology}

In the current ATLAS Inner Tracker, the silicon sensors are fabricated using the p-in-n technology. For the ITk, the strips are AC-coupled with n-type implants in a p-type float-zone silicon bulk $\left(\mathrm{n}^{+}\right.$-in-p FZ). Inter-strip isolation is achieved by $\mathrm{p}$-stop implants, uniform $\mathrm{p}$-spray, or a combination 
of both. This choice is motivated by the advantages that the $\mathrm{n}^{+}$-in-p technology offers over the p-in-n. First and foremost, the collected charges are electrons, faster and less subject to trapping: in the range of the expected maximum fluence, $8-10 \times 10^{14} \mathrm{n}_{e q} / \mathrm{cm}^{2}$, the $\mathrm{n}^{+}$-in-p sensors can deliver twice more charge. The process is single-sided, thus relatively easier and reasonably priced, with more foundries available worldwide. Figure 3 shows a cross section of the sensor, whose target thickness is between $300-320 \mu \mathrm{m}$.

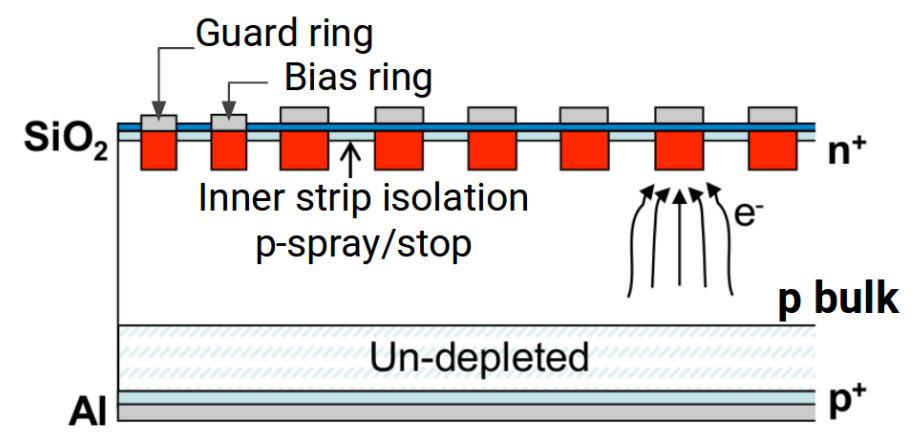

Figure 2: Schematic cross section of the ITk strip silicon sensors using $\mathrm{n}^{+}$-in-p technology.

The baseline design of the silicon sensor is based on 6-inch wafer technology. In the barrel the sensors are rectangular, with an active area of $96.640 \times 96.669 \mathrm{~mm}^{2}$. The two inner layers of the barrel (see Figure 1) are equipped with short strips of $24.1 \mathrm{~mm}$ length. The two outer layers have longer strips with $48.2 \mathrm{~mm}$. All strips in the barrel section have a pitch of $75.5 \mu \mathrm{m}$. In the end-cap the sensors are of trapezoidal shape, with all strips pointing towards the beam axis. With this geometry, the strips are radially oriented and provide the $\mathrm{R} \phi$ coordinate. The strip pitches range from 60 to $80 \mu \mathrm{m}$, while the strip lengths vary from 15 to $60 \mathrm{~mm}$, with the shortest ones chosen so as to balance the higher occupancy regions.

\subsection{Strip module concept}

To cover the large area of the silicon strip tracker, a large number of single units called "modules" will populate the surface of the new detector. As these modules will be massively produced (17,888 units), the process needs to be basic and low cost. A module consists of one sensor and one or two low-mass printed circuit boards, called hybrids, directly glued onto it. The hybrids host the read-out electronics and power distribution board. The strip sensors are connected to the front-end microelectronics ASIC binary chips via wire-bonds. An exploded schematic and a picture of a module can be seen in Figure 3. The same concept applies for both barrel and end-cap geometries, for either long or short strip versions.

\subsection{Signals and electronics}

A charged particle passing through the strip module creates a signal charge in the silicon sensor diode. This is converted to a hit by the closest ATLAS front-end chip (ABCStar), binary ASICs containing analogue preamplifier-shapers to process the signals from 256 strips. The schematic in Figure 4 gives an overview of the front-end electronics and power components, with three modules being represented. Each hybrid board can host up to 12 ABCStar ASICs, all connected to the 

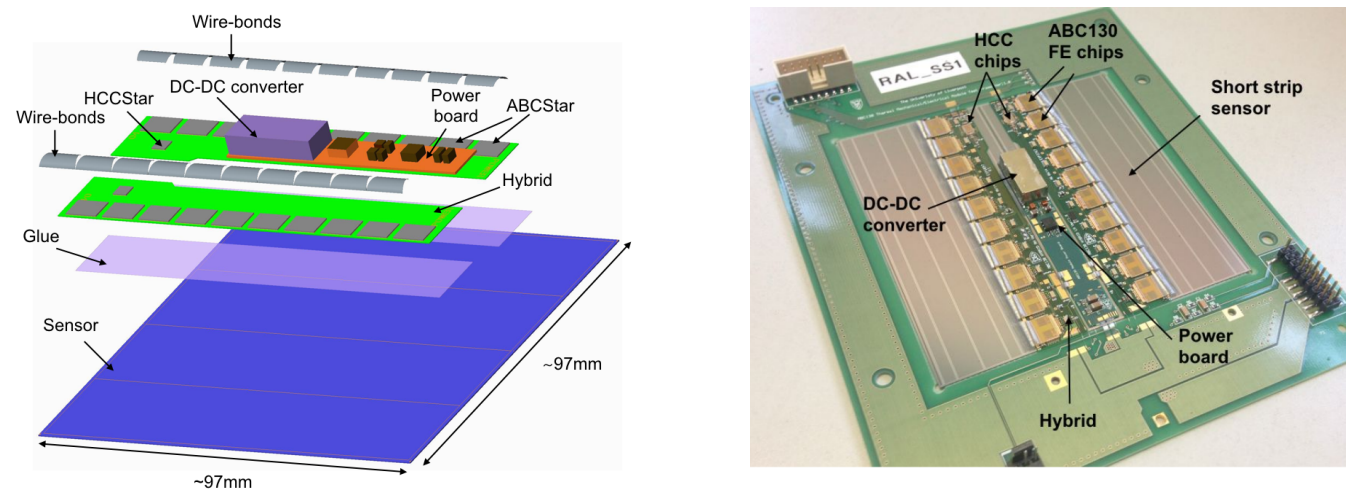

Figure 3: Left: exploded schematic of a module with the wire-bonds connecting the sensors to the front-end electronics and power board. The hybrid printed circuit board (green) is directly glued on the silicon strip sensors. Right: picture of an assembled module with the different components. Wire-bonds are visible in light grey at the edge of each ABC130 ASICs chips.

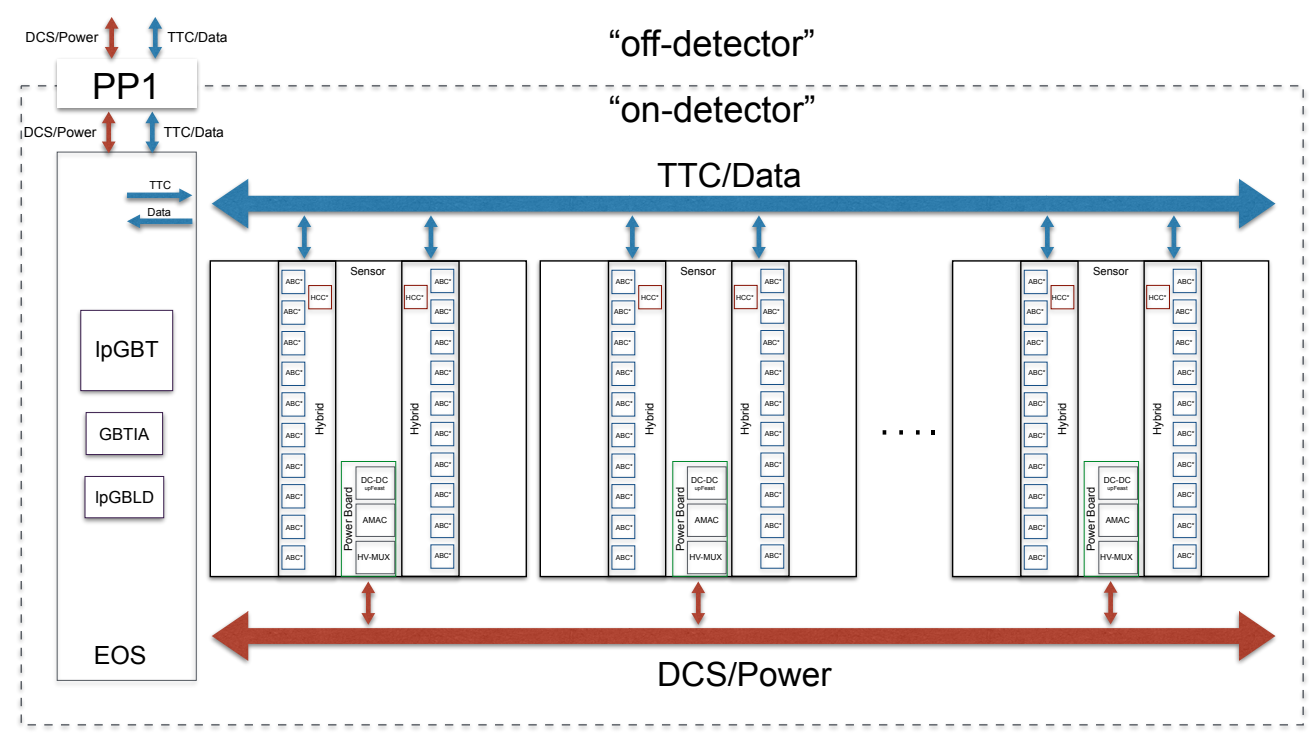

Figure 4: Overview of the electronics components of the ITk Strip Detector with three modules represented. Timing, Triggering and Control (TTC), power and DCS are interfaced to the stave/petal at the End-of-Substructure (EoS) card. 
Hybrid Controller Chip (HCCStar), which serves as an interface between the front-end electronics and the service bus. Both type of chips are planned to be produced in a $130 \mathrm{~nm}$ technology, as it features low power, high radiation tolerance, and high circuit density.

The HCCStar receives the clock and control signals (TTC) and distributes those to the ABCStars. The maximum data rate required is $160 \mathrm{Mbit} / \mathrm{s}$. In the other direction, the HCCStar builds packets of sensor data signals and send them via bus tapes to the so-called End-of-Substructure (EoS) card, at a rate of $640 \mathrm{Mbit} / \mathrm{s}$. The EoS collects in turn the sensor data, processes it and then transmits it via optical links to the data acquisition systems.

The distribution of power is performed through on-detector DC-DC converter modules. Each module is equipped with a power board containing three elements. The radiation hard buck converter ASIC “upFEAST", prototype currently under developement, receives a $10 \mathrm{~V}$ low-voltage power and generates the required $3.3 \mathrm{~V}$ and $1.5 \mathrm{~V}$ for each module. The autonomous monitor and control (AMAC) provides module level environmental measurements (current, voltage, temperature) and control (ABCStar, HHCStar, voltage switch, etc). The power board also hosts a High-Voltage Multiplexer (HV-MUX) to be able to connect or disconnect individual sensors from the high voltage bus. This provides module-level environmental measurements and control. Consequently, only a single power line is needed for a group of modules, reducing the material budget for services.

\subsection{Module support}

Modules are glued on local support structures. They consist of two face-sheets, which sandwich a core that is made from low-density carbon-fibre honeycomb. The core is sealed by glued close-outs. The cooling is ensured using a bi-phase $\mathrm{CO}_{2}$ at $-35^{\circ} \mathrm{C}$ circulated through titanium cooling pipes, which are surrounded by a high conductivity thermal foam, all embedded inside the sandwich structure. Figure 5 shows a conceptual cross section of the internal structure, common for both barrel and end-cap geometries.

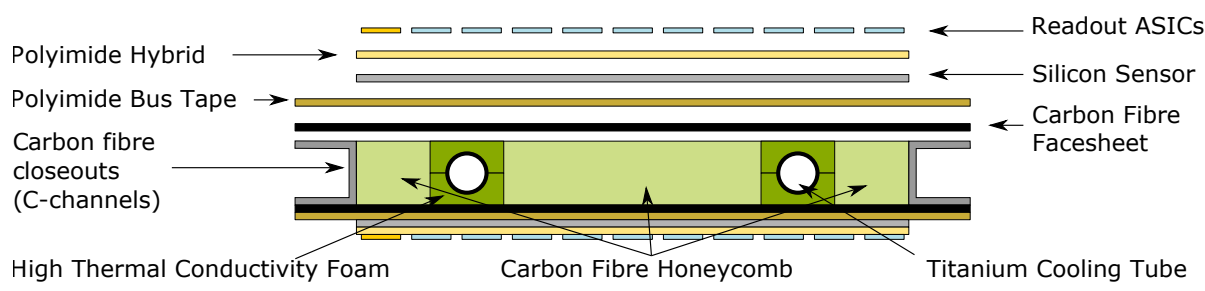

Figure 5: Schematic cross section of the module support arrangement, with the different layers exploded on the upper part. Both sides are populated with sensors. Although all elements will be glued, the glue layers are not shown for clarity.

This conceptual design leads to a thin structure, with a short thermal path $(\sim 3 \mathrm{~mm})$ and reduced radiation length $\left(\mathrm{x} / X_{0} \sim 2 \%\right)$. Material is thus minimized and the structure is kept relatively simple, advantageous for large scale reproducibility. The device is also modular, as the core and electronics components can be individually tested before assembly.

The local supports come in two types: the stave in the barrel and the petal in the end-caps. In the barrel, 14 rectangular modules are aligned on each side, forming the $1.4 \mathrm{~m}$ long stave unit, with the EoS on one end. In the end-cap, nine trapezoidal modules are arranged on each side of 
a wedge-shaped core, the so-called petal. Figure 6 illustrates the barrel and end-cap local support elements.
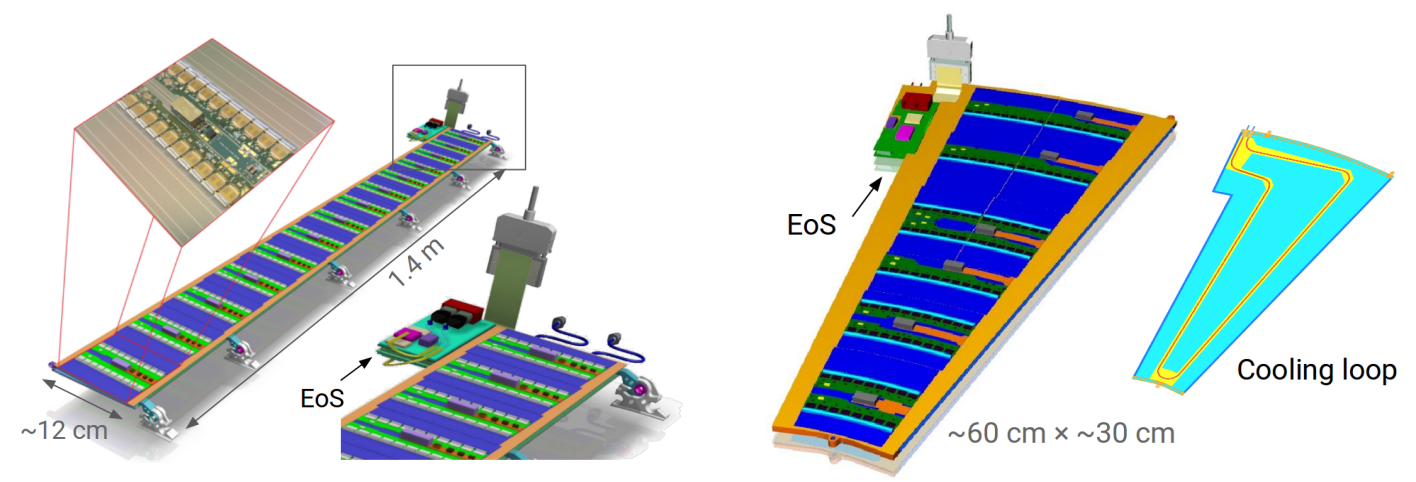

Figure 6: Schematic views of local support structures: the stave for the barrel (left) and the petal for the end-cap (right). Modules populate both sides of the support cores. The blue sensors are glued directly on the co-cured facing, drawn here in orange. The cooling pipe routing is shown at the right of the petal. The red lines indicate the titanium pipe; the yellow region shows the carbon-foam enclosing the pipes. The light blue area corresponds to the honeycomb filling material.

The staves and petals are mounted on global support structures, largely made of carbon-fibre. Their primary role is to locate precisely the substructures carrying the strip modules. The barrel consists of four concentric cylinders connected together at the ends of the cylinders by interlinks. The staves are inserted into the fully assembled cylinder structure from the ends, using temporary rails, as depicted in Figure 7. Petals are mounted onto carbon-fibre wheel structures, forming a disk, with 16 petals on both side. One end-cap contains six disks, as seen on the right of Figure 7. Figure 8 illustrates the large structures that constitute the ITk Detector.
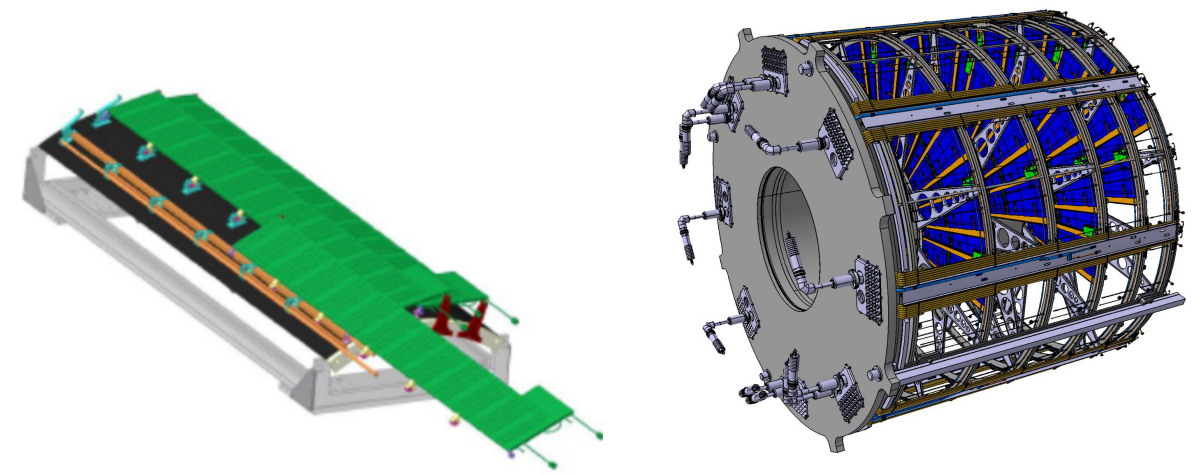

Figure 7: Left: barrel structure of the ITk detector, where staves are inserted with temporary rails. Right: the six end-cap disks, with the carbon-fiber holding structures. Some petals are inserted for illustration. 


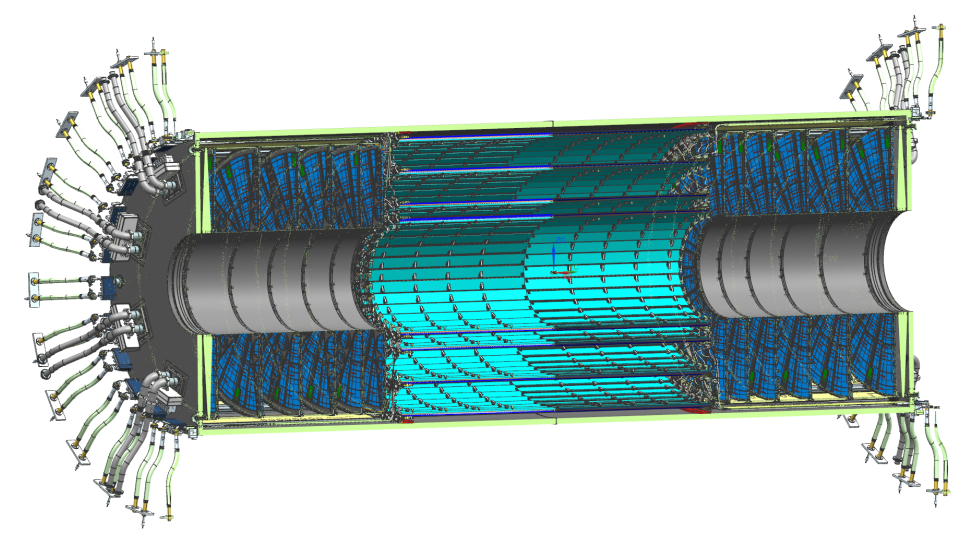

Figure 8: Schematic of the ITk Strip Detector. The four layers of staves aligned with the beam pipe, around the interaction point, form the barrel (in cyan), with the innermost layer being drawn entirely. Only the half cylinders of the three outward layers are visible for clarity. The two end-caps with inserted petals are drawn in blue. The outer cylinder (green rim) is closed by structural end-plates.

\section{Research and development}

Numerous studies are ongoing to optimise the design of the ITk Strip Detector, from its components to its overall layout, seeking the maximum performance. The following sections highlight some important results related to the silicon sensors and electronics.

\subsection{Total Ionizing Dose (TID) current increase}

An irradiation campaign with an X-ray source reveals an induced current increase of the prototype ABC130 binary ASIC chip. As the measured curves in Figure 9 clearly demonstrates, the increase is strongly temperature and dose rate dependent. This effect is well understood and typical of the $130 \mathrm{~nm}$ technology nodes [4].

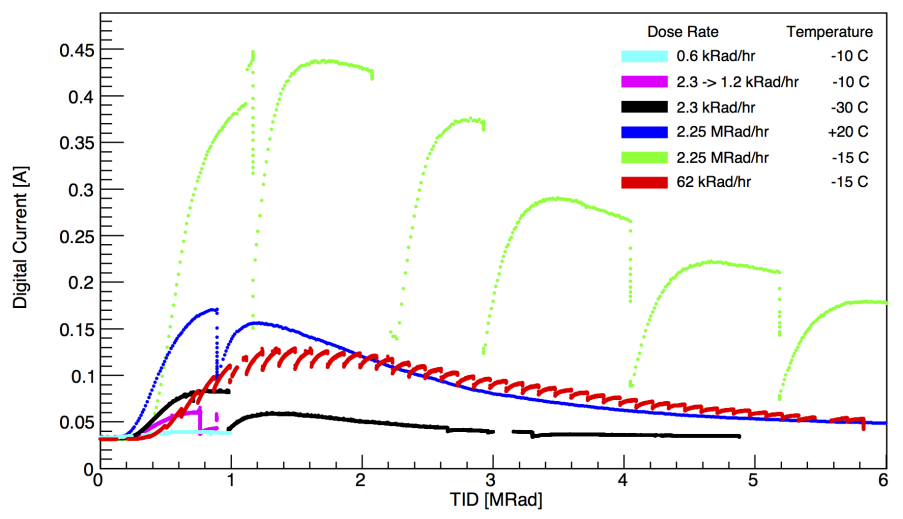

Figure 9: Digital current versus Total Ionizing Dose (TID) for ABC130 chips during X-rays irradiations at different dose rates and temperatures.

A detailed low dose rate irradiation campaign was conducted using the ${ }^{60} \mathrm{Co}$ source at CERN. For a temperature of $-25^{\circ} \mathrm{C}$ and a dose rate of $2 \mathrm{kRad} / \mathrm{h}$ (the highest expected dose for the ITk strips 
at the HL-LHC), the digital current increase reaches a factor of 2.5. Figure 10 shows the plotted measurements and a fit to these data points.

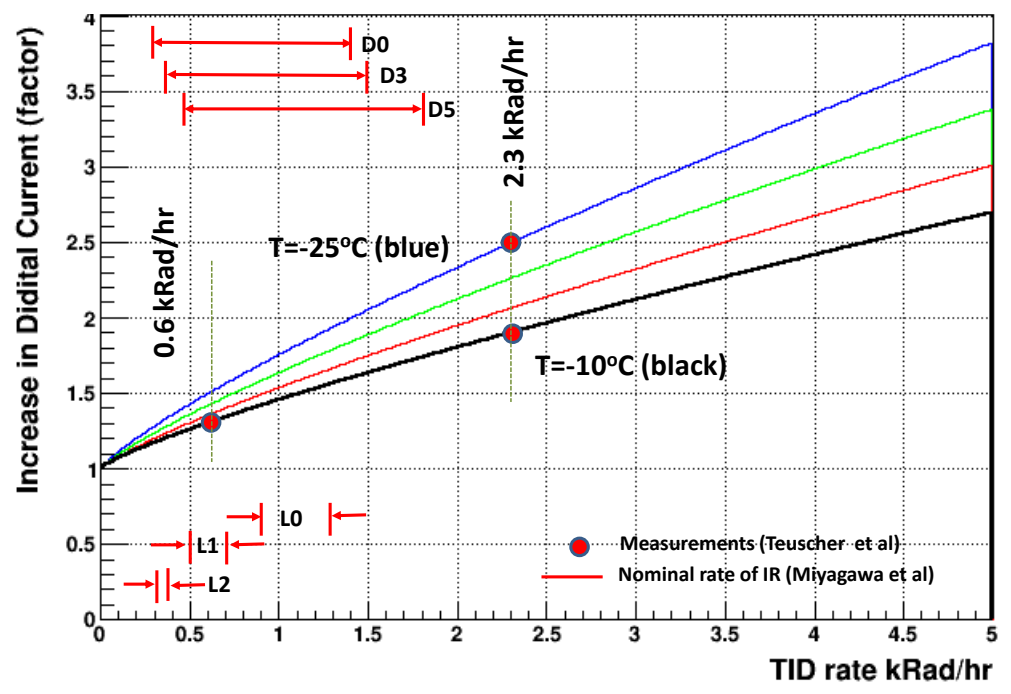

Figure 10: Current increase factor measured in $\mathrm{ABC} 130$ chips as a function of the dose rate for different temperatures.

Intensive studies are ongoing to characterise further the effect through additional irradiation tests. The dissipated power is modelled as a function of the fluence and the position. This information feeds the Finite Element Analysis (FEA) simulations to predict the digital current increase. The layout and architecture of the ITk Strip Detector is sturdy enough to incorporate this feature in the final design.

\subsection{Testbeam results}

Several test beam measurement campaigns have been performed at DESY and CERN in order to compare the performance of the sensors before and after radiation. In both facilities, the tracking was performed using the EUDET-style telescopes, see Figures 11a and 11b. An example of irradiated sensors is the Miniature Long Strip Barrel Module, shown in Figure 11c.

The efficiency curves were evaluated at DESY for the long-strip and short-strip regions. A follow-up testbeam at CERN allowed a comparison to be made between the performance of modules before and after irradiation. A single $130 \mathrm{~nm}$ long-strip barrel module (LS3) was irradiated at the CERN Proton Synchrotron with $24 \mathrm{GeV}$ protons. The received total ionising dose (TID) and nonionising energy loss damage were quantified to be $37.2 \mathrm{MRad}$ and $8 \times 10^{14} \mathrm{n}_{\mathrm{eq}} / \mathrm{cm}^{2}$ respectively. This corresponds to the maximum expected level of irradiation in the strip system, without applying any safety factors. Figure 12a shows the efficiency curves for long-strip regions of seven ASICs of the non-irradiated modules (LS4, in green) and for irradiated module (LS3, in red). As expected, the charge collection is reduced significantly after irradiation. However the non-irradiated modules exhibit an excellent efficiency plateau at $99 \%$ for threshold currents from 0.5 to $3 \mathrm{fC}$.

The expected end-of-lifetime performance has also been assessed. At the end-of-lifetime, modules are required to have a detection efficiency greater than $99 \%$ at thresholds that allow for 


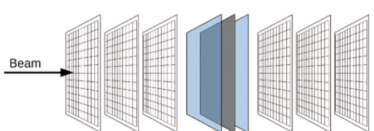

(a)

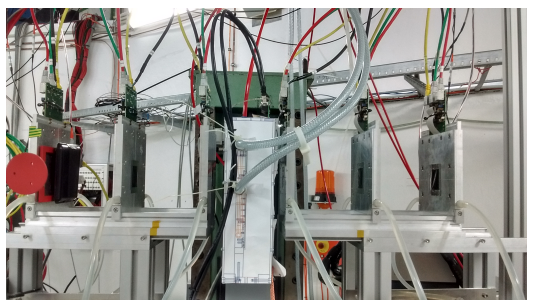

(b)

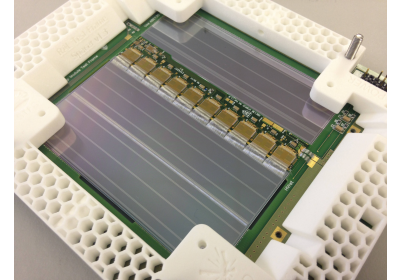

(c)

Figure 11: (a) Schematic of a EUDET-type telescope with the device under test between the six MIMOSA sensors. (b) The DURANTA Telescope at DESY with a barrel mini module under test. (c) Miniature Long Strip Barrel Module.

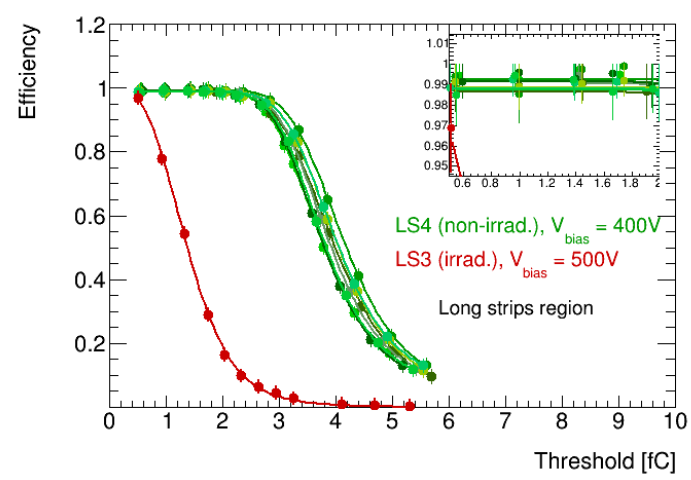

(a)

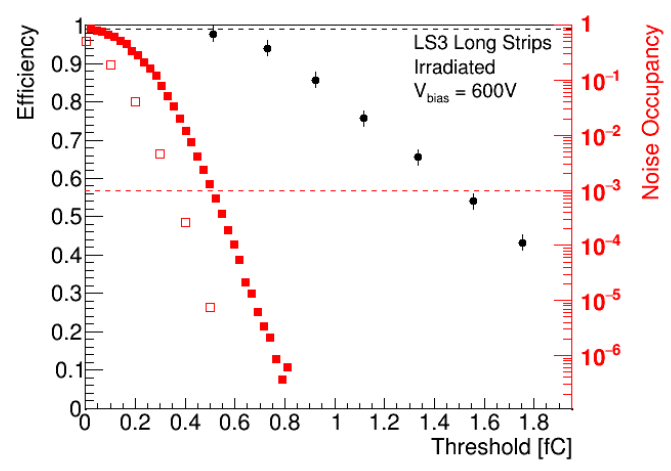

(b)

Figure 12: (a) The efficiency versus the threshold for four ASICs on the non-irradiated long-strip module LS4 (shown in green), and one ASIC on the irradiated module LS3 (shown in red). (b) The signal efficiency (black dots) compared to the noise occupancy at different thresholds for the $\mathrm{ABC} 130$ (red squares) and the new front-end (open squares). The results show the irradiated Long Strip Module 3 and a maximum bias voltage of $700 \mathrm{~V}$.

operation with less than $1 \times 10^{-3}$ channel noise occupancy. The noise after irradiation is the quadratic sum of the noise before irradiation and the shot noise after irradiation. The signal efficiency and noise occupancy are plotted in Figure 12b. With the prototype chip ABC130, the range of threshold currents for which both the signal efficiency and noise occupancy match the specifications above is extremely narrow. The measurement demonstrates however that for the reduced noise of the new front-end chip ABCStar, the range of thresholds that meets the specifications on noise occupancy and efficiency will be much wider.

\subsection{Prototype characterisation}

An intense effort has been pursued by the numerous institutes within the Strips ITk collaboration. Stave and petal prototypes are undergoing various tests, ranging from optical metrology, mechanical bending and stress, as well as thermal cycling for infrared measurements. Pictures of the current prototypes are shown in Figure 13. 


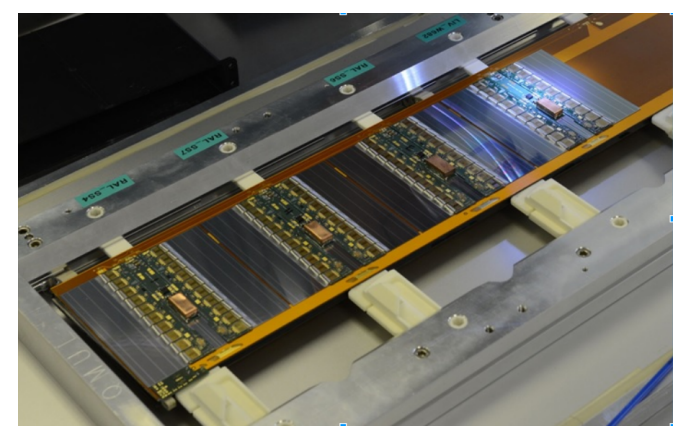

(a)

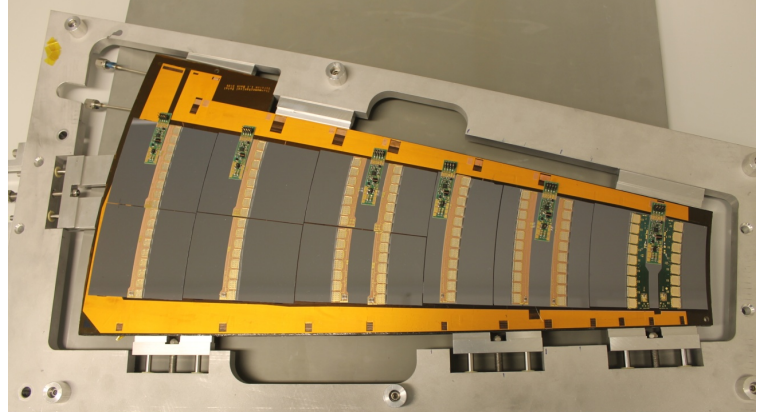

(b)

Figure 13: Photos of the thermo-mechanical stave (a) and petal (b) prototypes.

\section{Conclusion}

The design of the future Inner Tracker ITk of ATLAS Phase-II Upgrade is taking shape. It is aimed at meeting the requirements of the High-Luminosity LHC program, scheduled to start in 2026. The proposed all-silicon tracker system consists of a pixel detector at small radius close to the beam line and a large area strip tracker surrounding it. The HL-LHC will operate at an ultimate peak instantaneous luminosity up to $\mathcal{L}=7.5 \times 10^{34} \mathrm{~cm}^{-2} \mathrm{~s}^{-1}$ which corresponds to approximately 200 inelastic proton-proton collisions per beam crossing. The ITk will be operational for more than 10 years and in that time ATLAS aims for a total data set of $3000 \mathrm{fb}^{-1}$.

Numerous studies have been performed by the institutes of the Strips ITk collaboration in order to overcome the challenges of such a dense environment, striving to maximise performance. A strong prototyping effort is ongoing to optimise the stave and petal structures. Irradiation tests and test beam data taking campaigns show that the next generation of modules can withstand the radiation damage and match both the high signal efficiency and low noise occupancy requirements.

The results of these studies are summarized in the ATLAS Strips ITk Technical Design Report (TDR), published in May 2017. The Pixel TDR is expected for the end of 2017. The approval of this comprehensive document by the CERN Research Board in June 2017 counts as an important milestone on the road to 2026.

\section{References}

[1] ATLAS collaboration, The ATLAS Experiment at the CERN Large Hadron Collider, JINST 3 (2008) S08003.

[2] L. Evans and P. Bryant, LHC Machine, JINST 3 (2008) S08001.

[3] ATLAS collaboration, Technical Design Report for the ATLAS Inner Tracker Strip Detector, CERN-LHCC-2017-005. ATLAS-TDR-025, Apr, 2017.

[4] F. Faccio and G. Cervelli, Radiation-induced edge effects in deep submicron cmos transistors, IEEE Transactions on Nuclear Science 52 (Dec, 2005) 2413-2420. 